\title{
Sperm transport in the human female reproductive tract in relation to semen analysis characteristics and time of ovulation
}

\author{
D. Mortimer* and A. A. Templeton \\ Department of Obstetrics \& Gynaecology, University of Edinburgh, Centre for Reproductive \\ Biology, 37 Chalmers Street, Edinburgh EH3 9EW, U.K.
}

\begin{abstract}
Summary. Laparoscopic sperm recovery from the pouch of Douglas and tubal fimbriae was performed in 64 infertile couples. Spermatozoa were recovered from $16 / 35$ couples investigated after $\mathrm{AIH}$, and from 13/29 couples post coitum. The method of insemination had no effect on the result, which was positive in $45.3 \%$ of all couples, although AIH did result in significantly larger numbers of peritoneal spermatozoa. The number of peritoneal spermatozoa did not show any direct correlation with the number inseminated, but there were reductions along the tract of $5.83( \pm 1.14$ s.d.) orders of magnitude for total sperm count, and $5.52( \pm 1.21$ s.d.) for the number of motile spermatozoa. Only sperm motility had a significant influence on the success of sperm transport; spermatozoa were recovered from patients with sperm densities as low as 3.0 and $3.5 \times 10^{6} / \mathrm{ml}$, but with 56 and $44 \%$ motile spermatozoa. No influence of cycle day within the range \pm 4 days of ovulation on sperm transport was found. In 45 couples, routine semen analyses were apparently completely normal, but the incidence of sperm recovery was still only $49 \%$ (22/45), suggesting that a failure of sperm transport may have been a significant causative factor in their infertility.
\end{abstract}

\section{Introduction}

The physiology of sperm transport within the female reproductive tract of mammals has been extensively studied (Blandau, 1969; Bedford, 1972; Hafez, 1973, 1975, 1976; Ahlgren, Boström \& Malmqvist, 1974; Hunter, 1977; Moghissi, 1977a; Overstreet \& Katz, 1977; Mortimer, 1978) but reliable information for man is limited. Studies on human sperm transport are fraught with ethical and logistic problems, but knowledge of the physiology and pathology of this process is crucial to research on contraceptive and infertility problems.

In current clinical practice the only routine assessment of the interaction between spermatozoa and the female tract is the Sims-Hühner or post-coital test (see Hühner, 1924; Davajan, Nakamura \& Kharma, 1970). Unfortunately, this test suffers from a lack of standardization and its interpretation and prognostic value have been questioned (Jette \& Glass, 1972; Tredway et al., 1975; Asch, 1976, 1978; Moghissi, 1976, 1977b). The interaction between cervical mucus and spermatozoa has been evaluated in vitro (e.g. Kremer, 1965, 1968; Ulstein, 1972; Davajan \& Nakamura, 1973), but problems of standardization and interpretation still arise (see Matthews, Makin \& Cox, 1980). Correlations between sperm penetration of mucus

* Present address: Department of Obstetrics \& Gynaecology, University of Birmingham, Birmingham Maternity Hospital, Edgbaston, Birmingham B15 2TG, U.K. 
and semen parameters have been shown (e.g. Santomauro, Sciarra \& Varma, 1972; Ulstein, 1972; Moghissi, 1976; Matthews et al., 1980), but normal standards for semen analysis (Eliasson, 1971, 1975, 1977) are based on comparisons between ejaculates of fertile and suspected infertile men. Only one study to date has attempted to correlate semen parameters with the true success of sperm transport, i.e. the finding of spermatozoa at the site of fertilization (Ahlgren, 1969). Templeton \& Mortimer (1980) have shown that spermatozoa can be recovered from the peritoneal cavity of some infertile women after AIH. In this latter study there was a significantly higher incidence of subsequent pregnancies during a 1-year follow-up period in the group of women in whom spermatozoa were recovered.

The present study was an extension of this work to see if the success of laparoscopic sperm recovery could be related to semen characteristics, or to the time of coitus or artificial insemination with respect to ovulation.

\section{Patients and Methods}

\section{Patients}

Consenting couples were recruited from the Infertility Clinic, Royal Infirmary of Edinburgh. Prospective selection, of couples was based on: (1) normal medical history and physical examination of both partners; (2) regular (28 \pm 4 days), ovulatory cycles (confirmed by luteal-phase plasma progesterone measurement); and (3) semen analyses (usually 3) did not indicate sterility (Eliasson, 1977). Retrospective exclusions were made when significant pelvic pathology was found during the diagnostic laparoscopy and occasionally for technical problems with the sperm recovery (see Table 1).

Table 1. Summary of the patients investigated in the study and the numbers with successful laparoscopic sperm recovery

\begin{tabular}{|c|c|c|c|}
\hline & \multicolumn{2}{|c|}{ Insemination by: } & \multirow[b]{2}{*}{ Total } \\
\hline & AIH & Coitus & \\
\hline $\begin{array}{l}\text { No. of couples recruited } \\
\text { No. excluded }\end{array}$ & 49 & 39 & 88 \\
\hline Female pathology* & 9 & 6 & 15 \\
\hline Technical problems & 5 & 4 & 9 \\
\hline Actual study group & 35 & 29 & 64 \\
\hline
\end{tabular}

* Tubo-ovarian adhesions; endometriosis; congenital abnormality.

All couples agreed to abstain from intercourse for at least 3 days before investigation, and all laparoscopies were scheduled for midcycle (anticipated day of ovulation), estimated on the basis of previous menstrual data. Cycle timing was subsequently verified by integration of data from: (1) plasma LH, oestradiol and progesterone concentrations on (usually) the day before, day of, and day after surgery; (2) histological assessment of an endometrial biopsy; (3) dates of previous and next menstrual periods; and (4) visualization at laparoscopy of follicles or corpora lutea in the ovaries.

For patients having $\mathrm{AIH}$ the husband brought a masturbated semen specimen into the ward early on the morning of the day of surgery and this was inseminated (after semen analysis, see below) within $2 \mathrm{~h}$ of ejaculation. A standard clinical technique was used whereby a plastic catheter (Kwill: Everett Medical Products Ltd, Mitcham, Surrey, U.K.) was inserted into the external cervical os and the semen gently expelled. When coitus was the method of insemination patients were checked into hospital and then allowed home on the night before surgery. Patients were requested to time coitus depending on the scheduling of operating theatre lists. 


\section{Semen analysis}

For $\mathrm{AIH}$ patients, an aliquot $(\leqslant 0.5 \mathrm{ml})$ of the ejaculate was used for evaluation of sperm density, motility, viability and morphology by standard techniques (Eliasson, 1971, 1975); the ejaculate volume was measured with a graduated glass pipette. In couples using coital insemination semen data were obtained by taking average values of (usually) 3 ejaculates provided at monthly intervals just before the investigation. The term 'inseminate' is used to describe the semen deposited in the female tract, irrespective of the method of insemination. Further distinctions are used as necessary.

\section{Sperm recovery}

These procedures were performed at various times between 3.75 and $15 \mathrm{~h}$ after insemination according to the logistics of AIH or coitus and the scheduling of theatre lists. In all patients a trans-cervical flushing of the uterus with sterile medium (Medium 199 with Earle's salts and Hepes buffer: Flow Labs, Irvine, U.K.) was attempted using a 3-way balloon catheter (modified from Maathuis \& Aitken, 1978) before starting the diagnostic laparoscopy and its associated test of tubal patency using blue dye injected into the uterine cavity under low pressure. In addition to the routine procedures of a diagnostic laparoscopy for the assessment of the pelvic organs (Templeton \& Kerr, 1977), the fluid lying in the cul-de-sac or pouch of Douglas was aspirated using a blunt-ended needle $(2 \mathrm{~mm}$ diam.). The fimbriated end of each Fallopian tube was then separately rinsed with $10 \mathrm{ml}$ of the same medium and the rinsings aspirated from the pouch of Douglas. An endometrial biopsy, using a Sharman curette, was the last procedure.

\section{Processing of material}

In the laboratory, the volumes of all flushings were measured and from each a known fraction (usually about $1 / 10$ ) was taken for subsequent processing and scoring. During the course of the project ( 3 years) several improvements in the processing methods were made to facilitate the scoring of spermatozoa in the material recovered from the female tract. Each improvement was fully evaluated against its predecessor on duplicate aliquots of tract specimens or on artificially produced 'flushings'. The final method, used for the last $1 \frac{1}{2}$ years, was as follows.

The aliquot of each flushing was treated with twice its volume of $0.01 \%(\mathrm{w} / \mathrm{v})$ aqueous saponin solution (Saponin powder: Coulter Electronics, Harpenden, Herts, U.K.) to lyse contaminating red blood cells, centrifuged at $500 \mathrm{~g}$ for $10 \mathrm{~min}$, and the pellet resuspended in 1.0 $\mathrm{ml}$ fresh saponin solution. After a second centrifugation under the same conditions the pellet was resuspended in $15 \mu$ distilled water and the suspension spotted onto a clean microscope slide as 3 equal ( 6 or $7 \mu$ ) droplets. Slides were dried overnight in a $37^{\circ} \mathrm{C}$ incubator, fixed in absolute ethanol and allowed to air dry. Rings were drawn around the area of each drop on the underside of the slides using a spirit-based marker pen to facilitate location of the sample material after mounting. Glycerine jelly (Raymond A. Lamb, London, U.K.), melted at $60^{\circ} \mathrm{C}$, was used to mount the preparations.

For scoring, the entire area of each drop was scanned under phase-contrast optics at magnifications ranging from $\times 250$ to $\times 500$ (dependent upon the amount of residual background material and on the microscope used). The numbers of spermatozoa present were used to calculate the total number present in the whole flushing.

\section{Results}

The success of laparoscopic sperm recovery, i.e. the finding of spermatozoa in one or more of the pouch of Douglas aspirate or fimbrial rinses, was not significantly different between the AIH and coital couples, and gave an overall success rate of $45 \%$ (Table 1 ). 


\section{Uterine flushings}

Failure of the uterine flushing technique (Table 2) was, in all cases, due to the inability to obtain a 'seal' on the cervix when the balloon was inflated with warm saline, resulting in expulsion of the balloon region of the catheter from the cervical canal. Flushings were obtained from over half of the patients. There was no significant difference between the two groups for successful flushing but mucoid material was more often present in the flushings from the post-coital patients $(P<0.001)$. Such samples were discarded because the finding of mucus was taken as indicating probable contamination with cervical material.

Table 2. Summary of the results obtained using trans-cervical

flushing as a method for recovering uterine spermatozoa

\begin{tabular}{lccc}
\hline & \multicolumn{2}{c}{ Insemination by: } & \\
\cline { 2 - 3 } & AIH & Coitus & Total \\
\hline Study group & 35 & 29 & 64 \\
Flushing not possible (\%) & 12 & 15 & $27(42 \cdot 2)$ \\
Flushings obtained (\%) & 23 & 14 & $37(57 \cdot 8)$ \\
Contaminated with mucus & $1^{*}$ & $11^{*}$ & 12 \\
Uterine sample obtained (\%) & 22 & 3 & $25(37 \cdot 5)$ \\
Sperm positive & 15 & 1 & 16 \\
\hline
\end{tabular}

*Significantly different $\left(P<0.001,2 \times 2 \chi^{2}\right.$ test $)$.

Spermatozoa were reliably recovered from the uterine lumen of $25 \%$ of all patients investigated and $64 \%$ of those patients from whom an acceptable uterine sample had been obtained. The estimated total numbers of spermatozoa found in the uterine lumen ranged from 68 to 21083 , with 9 zero values. In the sperm-positive samples the mean total number was 4743 ( \pm 6900 s.d.).

\section{Pouch of Douglas and fimbrial material}

The results for sperm recovery in relation to the day of ovulation are shown in Table 3 . Precise determination of the day of ovulation was not always possible (see 'Methods') but because one of the criteria for recruitment of patients was a reasonably regular menstrual cycle, the patients classed as being $\pm \geqslant 2$ days with respect to ovulation would be unlikely to be $> \pm 4$ days. There were no significant differences ( $\chi^{2}$ tests) in the success rates for sperm recovery

Table 3. Incidence of positive laparoscopic sperm recovery (+, positive; - , negative) in relation to the timing of the procedure with respect to the day of ovulation

\begin{tabular}{|c|c|c|c|c|c|c|}
\hline \multirow{3}{*}{$\begin{array}{l}\text { Relative to day } \\
\text { of ovulation }\end{array}$} & \multicolumn{4}{|c|}{ Insemination by: } & & \\
\hline & \multicolumn{2}{|c|}{ AIH } & \multicolumn{2}{|c|}{ Coitus } & \multicolumn{2}{|c|}{ Total } \\
\hline & + & - & + & - & + & - \\
\hline$\geqslant-2$ & 7 & 8 & 2 & 7 & 9 & 15 \\
\hline$<-2$ & 3 & 4 & 5 & 2 & 8 & 6 \\
\hline 0 & 2 & 2 & 2 & 3 & 4 & 5 \\
\hline$<+2$ & 3 & 2 & 0 & 2 & 3 & 4 \\
\hline$\geqslant+2$ & 1 & 3 & 4 & 2 & 5 & 5 \\
\hline Total & 16 & 19 & 13 & 16 & 29 & 35 \\
\hline
\end{tabular}

* See 'Methods' for timing. 
between the various times within the AIH and coital patients, or between $\mathrm{AIH}$ and coitus as the means of insemination. When the two groups were combined there were still no differences between the timing groups. It was therefore considered that all patients could be taken as a single population for the analysis of the influence of semen analysis parameters on the success of sperm transport (Text-fig. 1).

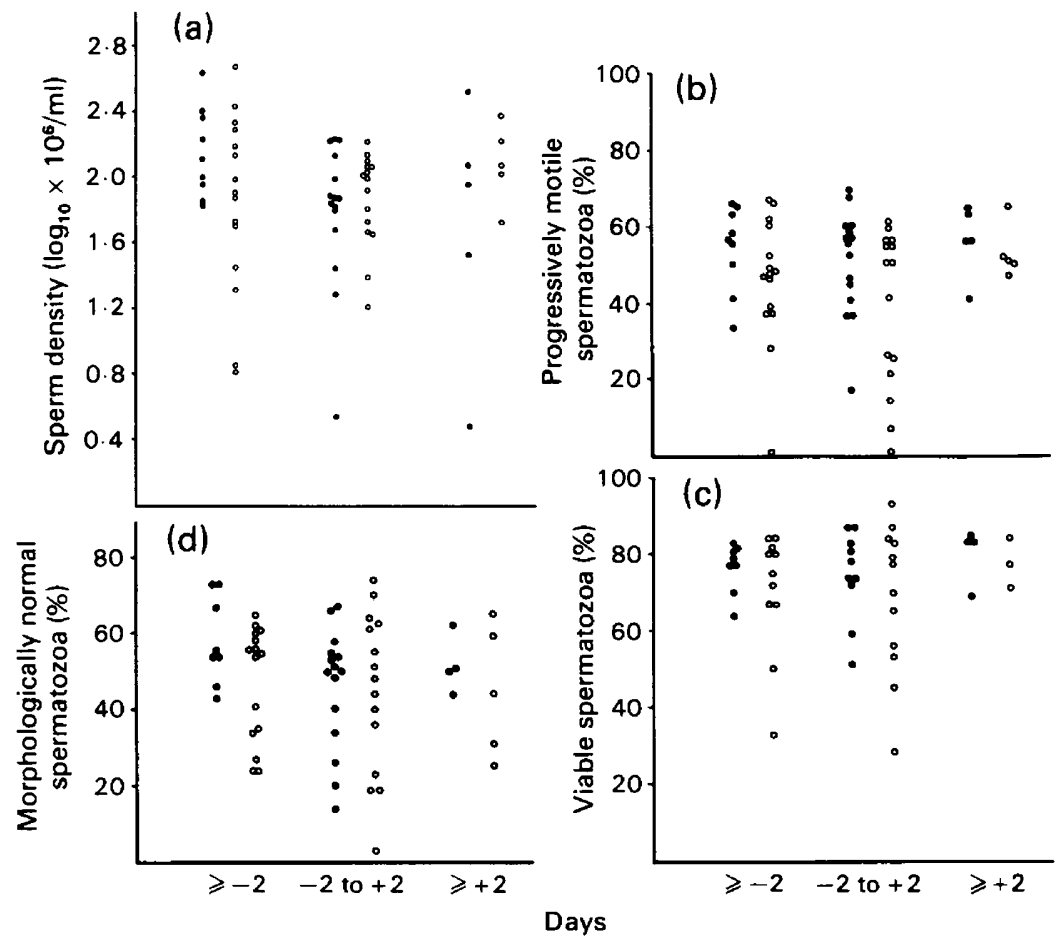

Text-fig. 1. The distribution of sperm positive (O) and negative $(0)$ patients in relation to the day of ovulation (see text) and (a) $\log _{10}$ sperm density in the inseminate, (b) \% progressively motile spermatozoa, (c) \% viable (non-eosinophilic) spermatozoa, and (d) \% morphologically normal spermatozoa in the inseminate (combined data from $\mathrm{AIH}$ and coital tests).

Table 4. Semen analysis characteristics and the result of laparoscopic sperm recovery

\begin{tabular}{lcc}
\hline & \multicolumn{2}{c}{ Laparoscopic sperm recovery } \\
\cline { 2 - 3 } \multicolumn{1}{c}{ Semen characteristics } & Positive* & Negative* $^{*}$ \\
\hline Volume $(\mathrm{ml})$ & $3.3 \pm 1.9$ & $2.9 \pm 1.4$ \\
Sperm density $\left(\times 10^{6} / \mathrm{ml}\right)$ & $113.5 \pm 93.3$ & $106.6 \pm 87.6$ \\
Log $_{10}$ sperm density $\left(\times 10^{6} / \mathrm{ml}\right) \ddagger$ & $1.88 \pm 0.48$ & $1.88 \pm 0.41$ \\
Square root sperm density $\left(\times 10^{6} / \mathrm{ml}\right) \ddagger$ & $9.81 \pm 4.24$ & $9.56 \pm 3.96$ \\
Total sperm count $\left(\times 10^{6} / \mathrm{ejaculate}\right)$ & $335.6 \pm 287.6$ & $282.5 \pm 225.3$ \\
Motility (\% progressive) & $52.4 \pm 12.2 \dagger$ & $43.6 \pm 17.9 \dagger$ \\
Viability (\% non-eosinophilic) & $76.3 \pm 9.2^{\mathrm{a}}$ & $70.2 \pm 16.7^{\mathrm{b}}$ \\
Morphology (\% normal forms) & $50.9 \pm 14.5^{\mathrm{c}}$ & $45.9 \pm 17.7$ \\
\hline
\end{tabular}

*Values are mean \pm s.d., number of tests $=29$ positive and 35 negative except where indicated otherwise by superscript letters: ${ }^{\mathrm{a}}=22,{ }^{\mathrm{b}}=26,{ }^{\mathrm{c}}=28$.

†Significantly different, $P<0.025$ (Student's $t$ test).

$\ddagger$ Transformations attempting to normalize the distribution. 
A significant $(P<0.025)$ difference between patients exhibiting positive or negative sperm recovery was found only in respect of the percentage of progressively motile spermatozoa (Table 4). Two severely oligozoospermic patients $\left(3.0\right.$ and $\left.3.5 \times 10^{6} / \mathrm{ml}\right)$ showed positive sperm recovery (see Text-fig. 1a); the percentages of progressive spermatozoa for these two patients were 56 and $44 \%$ respectively.

There were no significant relationships between sperm transport success, proximity to ovulation, and various semen analysis parameters, although there were clear indications that sperm recovery was more likely for poorer semen quality if insemination occurred near to the time of ovulation (Text-fig. 1). This was particularly clear for sperm morphology (Text-fig. 1d), but the groups were too small to permit adequate statistical analysis.

No correlations were found between the numbers of peritoneal spermatozoa and either the total number or the number of motile spermatozoa artificially or coitally inseminated (using actual or $\log _{10}$ numbers). The numbers of peritoneal spermatozoa recovered (Text-fig. 2) were significantly greater after AIH than after coitus (Wilcoxon Rank Sum test, $P<0.001$ ). This difference could not be explained on the grounds of more spermatozoa accumulating in the pouch of Douglas with increasing time after insemination because all the AIH patients were investigated after the shorter time interval of 4-8 h. A Wilcoxon Rank Sum test showed that the numbers of peritoneal spermatozoa recovered in the coitally inseminated patients were unrelated to the time of laparoscopy post coitum (4-8 or 12-16 h).

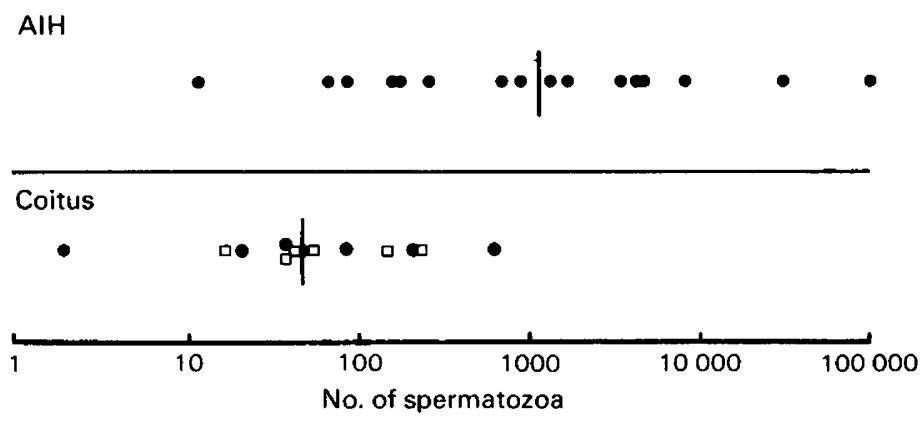

Text-fig. 2. Numbers of peritoneal spermatozoa recovered at laparoscopy 4-8 h (O) or 12-16 h (ㅁ) after insemination in patients inseminated artificially or at coitus. Vertical bars denote medians: 1162 (range 12-100 563) for 16 AIH patients, 49 (range 2-660) for 13 coital patients, and 175 (range $2-100563$ ) for all 29 patients.

\section{Discussion}

The finding of spermatozoa in the peritoneal cavity of $45 \%$ of patients in the present study (Table 2) is higher than that reported for the only other two reported equivalent large studies (Ahlgren, 1969: 53/177 = 30\%; Hammerstein, Zielske, Kratzch \& Koch, 1977: 104/365 = 29\%). Croxatto et al. (1974) and Asch (1976) obtained values of 4/6 (67\%) and 9/11 (82\%), but these were only small studies and involved selected patients. The studies of Ahlgren (1969) and Hammerstein et al. (1977) included patients inseminated at all stages of the menstrual cycle, and both found a significant impairment of sperm transport during the early follicular and the luteal phases. The present study, however, concentrated on the periovulatory period (midcycle \pm 4 days), during which the conditions for sperm transport are optimum. There were no differences in sperm recovery rates over this period. The numbers of spermatozoa recovered from the uterine lumen are in general agreement with the range reported by Croxatto et al. (1974) for a similar time period after insemination, although the present range is somewhat wider at the upper limit. 
The trans-cervical route was not found to be a useful method for obtaining flushings of the uterine lumen that were reliably free from contamination with cervical material. Although it is possible that the mucoid material found in many of the uterine flushes may have been of uterine origin, it was impossible to determine its source.

That artificial insemination into the external os increases the number of spermatozoa transported through the human female tract has not hitherto been reported. Physiologically it is not unexpected, but it emphasizes that studies on sperm transport may well be influenced by the use of artificial insemination. However, there was no evidence to suggest that false-positive sperm recovery results were created by this procedure because the values were identical for both groups of patients (see Table 1).

The absence of correlations between the number of spermatozoa transported through the tract and the number inseminated is apparently at variance with the findings of Settlage, Motoshima \& Tredway (1973, 1974). Although this could be explained by the great intrinsic variability of sperm numbers, it could also be due to technical differences. Settlage et al. (1973, 1974) investigated spermatozoa recovered from excised Fallopian tubes, where a constant population of spermatozoa may be maintained (see Overstreet \& Katz, 1977). The accumulation of greater numbers of spermatozoa in the pouch of Douglas could result from a continual passage of spermatozoa through the tract into the peritoneal cavity. Furthermore, the patients studied by Settlage et al. (1974) were highly selected for excellent semen quality. Our data do, however, support the general conclusion (Ahlgren et al., 1974; Settlage et al., 1974) that there is usually a reduction in sperm numbers along the length of the female tract of between 5 and 6 orders of magnitude: $5.83 \pm 1.14$ s.d. for total numbers, and $5.52 \pm 1.21$ s.d. for motile spermatozoa inseminated.

Although Ahlgren (1969) found no significant differences between semen analysis parameters and the success of sperm transport, we did find a difference for the percentage of progressively motile spermatozoa (see Table 4). Sperm motility is clearly the most important parameter for a prognosis of successful sperm transport (although such spermatozoa must also be intrinsically fertile). Sperm density, a parameter often used in isolation to describe a man's fertility potential, has no clear prognostic value for sperm transport success. Even when all the characteristics of the routine semen analysis were apparently normal (45 cases) there was still only a $49 \%$ chance of spermatozoa being found in the peritoneal cavity.

Although there is always the possibility of falsely negative tests, the results of Templeton \& Mortimer (1980), showing that for patients from whom spermatozoa were not recovered, $0 / 9$ conceived during a 1-year follow-up period, would indicate that many, if not all, of such patients had genuine failure of sperm transport. Impaired sperm transport could therefore be a significant causative factor in infertility, but could be due to the spermatozoa or the female tract.

The technique of laparoscopic sperm recovery need add only about $5 \mathrm{~min}$ to the routine diagnostic laparoscopy procedure, and, when correctly scheduled, can provide physiologically meaningful information on the interaction between spermatozoa and the female tract.

D.M. was supported by a Wellcome Trust Interdisciplinary Linked Fellowship which also supported the technical assistance of Mrs E. Leslie, and A.A.T. was in receipt of a Wellcome Trust Surgical Fellowship during the initial stage of the project. We thank Mr L. P. Mackenzie, Mrs C. Reid and Mr K. Donachie for further technical assistance, the staff of the Edinburgh Royal Infirmary Infertility Clinic, and Ms R. Carpenter for typing the manuscript.

\section{References}

Ahlgren, M. (1969) Migration of spermatozoa to the Fallopian tubes and abdominal cavity in women including some immunological aspects. M.D. Thesis, University of Lund, Sweden, p. 139.
Ahlgren, M., Boström, K. \& Malmqvist, R. (1974) Sperm transport and survival in women with special reference to the Fallopian tube. In Sperm Transport, Survival and Fertilizing Ability in Vertebrates, Vol. 
26, pp. 183-200. Eds E. S. E. Hafez \& C. G. Thibault. INSERM, Paris.

Asch, R.H. (1976) Laparoscopic recovery of sperm from peritoneal fluid, in patients with negative or poor Sims-Huhner test. Fert. Steril. 27, 1111-1114.

Asch, R.H. (1978) Sperm recovery in peritoneal aspirate after negative Sims-Huhner test. Int. J. Fert. 23 , $57-60$.

Bedford, J.M. (1972) Sperm transport, capacitation and fertilization. In Reproductive Biology, Ch. 6, pp. 338-392. Eds H. Bakin \& S. R. Glasser. Excerpta Medica, Amsterdam.

Blandau, R.J. (1969) Gamete transport-comparative aspects. In The Mammalian Oviduct. Comparative Biology and Methodology, pp. 129-162. Eds E. S. E. Hafez \& R. J. Blandau. University of Chicago Press, Chicago.

Croxatto, H.B., Faundes, A., Medel, M., Avendaño, S., Croxatto, H.D., Vera, C., Anselmo, J. \& Pastene, L. (1974) Studies on sperm migration in the human female genital tract. In Sperm Transport, Survival and Fertility Ability in Vertebrates, Vol. 26, pp. 162-182. Eds E. S. E. Hafez \& C. G. Thibault. INSERM, Paris.

Davajan, V. \& Nakamura, R.M. (1973) The in vitro sperm-cervical mucus testing systems. In Cervical Mucus in Human Reproduction. Ch. 12, pp. 153161. Eds M. Elstein, K. S. Moghissi \& R. Borth. Scriptor, Copenhagen.

Davajan, V., Nakamura, R.M. \& Kharma, K. (1970) Spermatozoan transport in cervical mucus. Obstet. gynec. Surv. 25, 1-43.

Eliasson, R. (1971) Standards in evaluation of human semen. Andrologie 3, 49-64.

Eliasson, R. (1975) Analysis of semen. In Progress in Infertility, 2nd edn), Ch. 33, pp. 691-713. Eds S. J. Behrman \& R. W. Kistner. Little, Brown \& Co., Boston.

Eliasson, R. (1977) Semen analysis and laboratory workup. In Male Infertility, Workup. Treatment and Research, pp. 169-188. Eds A. T. K. Cockett \& R. L. Urry. Grune \& Stratton, New York.

Hafez, E.S.E. (1973) Gamete transport. In Human Reproduction, Conception and Contraception, Ch. 5, pp. 85-118. Eds E. S. E. Hafez \& T. N. Evans. Harper \& Row, New York.

Hafez, E.S.E. (1975) Sperm transport. In Progress in Infertility, 2nd edn, Ch. 7, pp. 143-174. Eds S. J. Behrman \& R. W. Kistner. Little, Brown \& Co., Boston.

Hafez, E.S.E. (1976) Transport and survival of spermatozoa in the female reproductive tract. In Human Semen and Fertility Regulation in Men, Ch. 11 , pp. 107-129. Ed. E. S. E. Hafez. C. V. Mosby Co., Saint Louis.

Hammerstein, J., Zielske, F., Kratzch, E. \& Koch, U.J. (1977) Sperm migration throughout the female genital tract in relation to the time of ovulation. In The Uterine Cervix in Reproduction, pp. 238-242. Eds V. Insler \& G. Bettendorf. Georg Thieme Publishers, Stuttgart.

Hühner, M. (1924) The diagnosis of sterility in the male and the female. Am. J. Obstet. Gynec. 8, 63-75.

Hunter, R.H.F. (1977) Function and malfunction of the
Fallopian tubes in relation to gametes, embryos and hormones. Eur. J. Obstet. Gynec. Reprod. Biol. 7, 267-283.

Jette, N.T. \& Glass, R.H. (1972) Prognostic value of the post-coital test. Fert. Steril. 23, 29-32.

Kremer, J. (1965) A simple sperm migration test. Int. J. Fert. 10, 209-215.

Kremer, J. (1968) The in vitro spermatozoal penetration test in fertility investigations. Ph.D. dissertation, University of Groningen, The Netherlands.

Maathuis, J.B. \& Aitken, R.J. (1978) Cyclic variation in concentrations of protein and hexose in human uterine flushings collected by an improved technique. J. Reprod. Fert. 52, 289-295.

Matthews, C.D., Makin, A.F. \& Cox, L.W. (1980) Experience with in vitro sperm penetration testing in infertile and fertile couples. Fert. Steril. 33, 187-192.

Moghissi, K.S. (1976) Postcoital test: physiologic basis, technique and interpretation. Fert. Steril. 27, 117129.

Moghissi, K.S. (1977a) Sperm migration through the human cervix. In The Uterine Cervix in Reproduction, pp. 146-165. Eds V. Insler \& G. Bettendorf. Georg Thieme Publishers, Stuttgart.

Moghissi, K.S. (1977b) Significance and prognostic value of post-coital test. In The Uterine Cervix in Reproduction, pp. 231-238. Eds V. Insler \& G. Bettendorf. Georg Thieme Publishers, Stuttgart.

Mortimer, D. (1978) Selectivity of sperm transport in the female genital tract. In Spermatozoa, Antibodies and Infertility, Ch. 5, pp. 37-53. Eds J. Cohen \& W. F. Hendry. Blackwell Scientific Publications, Oxford.

Overstreet, J.W. \& Katz, D.F. (1977) Sperm transport and selection in the female genital tract. In Development in Mammals, Vol. 2, pp. 31-65. Ed. M. H. Johnson. North Holland, Amsterdam.

Santomauro, A.G., Sciarra, J.J. \& Varma, A.O. (1972) A clinical investigation of the role of the semen analysis and post-coital tests in the evaluation of male infertility. Fert. Steril. 23, 245-251.

Settlage, D.S.F., Motoshima, M. \& Tredway, D.R. (1973) Sperm transport from the external cervical os to the Fallopian tubes in women: a time and quantitation study. Fert. Steril. 24, 655-661.

Settlage, D.S.F., Motoshima, M. \& Tredway, D.R. (1974) Sperm transport from the external os to the Fallopian tubes in women: a time and quantitative study. In Sperm Transport, Survival and Fertilizing Ability in Vertebrates, Vol. 26, pp. 210-217. Eds E. S. E. Hafez \& C. G. Thibault. INSERM, Paris.

Templeton, A.A. \& Kerr, M.G. (1977) An assessment of laparoscopy as the primary investigation in the subfertile female. Br. J. Obstet. Gynaec. 84, 760-762.

Templeton, A.A. \& Mortimer, D. (1980) Laparoscopic sperm recovery in infertile women. Br.J. Obstet. Gynaec. 87, 1128-1131.

Tredway, D.R., Settlage, D.S., Nakamura, R.M., Motoshima, M.L., Umezaki, C.U. \& Mishell, D.R. (1975) Significance of timing for the postcoital evaluation of cervical mucus. Am. J. Obstet. Gynec. 121, 387-393.

Ulstein, M. (1972) Sperm penetration of cervical mucus as a criterion of male fertility. Acta obstet. gynec. scand. 51, 335-340. 